MATHEMATICS OF COMPUTATION

Volume 71, Number 240, Pages 1641-1661

S 0025-5718(01)01377-1

Article electronically published on August 2, 2001

\title{
THE ERROR BOUNDS AND TRACTABILITY OF QUASI-MONTE CARLO ALGORITHMS IN INFINITE DIMENSION
}

\author{
FRED J. HICKERNELL AND XIAOQUN WANG
}

\begin{abstract}
Dimensionally unbounded problems are frequently encountered in practice, such as in simulations of stochastic processes, in particle and light transport problems and in the problems of mathematical finance. This paper considers quasi-Monte Carlo integration algorithms for weighted classes of functions of infinitely many variables, in which the dependence of functions on successive variables is increasingly limited. The dependence is modeled by a sequence of weights. The integrands belong to rather general reproducing kernel Hilbert spaces that can be decomposed as the direct sum of a series of their subspaces, each subspace containing functions of only a finite number of variables. The theory of reproducing kernels is used to derive a quadrature error bound, which is the product of two terms: the generalized discrepancy and the generalized variation.

Tractability means that the minimal number of function evaluations needed to reduce the initial integration error by a factor $\varepsilon$ is bounded by $C \varepsilon^{-p}$ for some exponent $p$ and some positive constant $C$. The $\varepsilon$-exponent of tractability is defined as the smallest power of $\varepsilon^{-1}$ in these bounds. It is shown by using Monte Carlo quadrature that the $\varepsilon$-exponent is no greater than 2 for these weighted classes of integrands. Under a somewhat stronger assumption on the weights and for a popular choice of the reproducing kernel it is shown constructively using the Halton sequence that the $\varepsilon$-exponent of tractability is 1 , which implies that infinite dimensional integration is no harder than onedimensional integration.
\end{abstract}

\section{INTRODUCTION}

The evaluation of complicated integrals is a common computational problem occurring in many areas of sciences such as computational physics, statistics, computer graphics and mathematical finance. The univariate case is well-developed DR84, and the multivariate case has been extensively studied in recent years (see CMO97, FW94, Hic98, Nie92, S.J94, SW98, Sob98, Woź91 and the references therein).

For high dimensional integration, $I_{s}(f)=\int_{[0,1]^{s}} f(\mathbf{x}) d \mathbf{x}$, Monte Carlo (MC) and quasi-Monte Carlo (QMC) methods can be used to break the curse of dimensionality. $\mathrm{MC}$ and $\mathrm{QMC}$ use the sample mean, $Q_{N, s}(f ; P)=\frac{1}{N} \sum_{\mathbf{z} \in P} f(\mathbf{z})$, to

Received by the editor May 24, 2000 and, in revised form, October 18, 2000.

2000 Mathematics Subject Classification. Primary 65C05, 65D30.

Key words and phrases. Quasi-Monte Carlo methods, Monte Carlo methods, tractability, infinite dimensional integration.

This work was supported by a Hong Kong Research Grants Council grant RGC/97-98/47 and by the NSF of China Grants 79970120 and 10001021. 
approximate the integral $I_{s}(f)$. For MC, the sample points $P$ are independent random points distributed uniformly on $[0,1]^{s}$, and the asymptotic convergence rate is $O\left(N^{-1 / 2}\right)$, which is independent of the dimension. For QMC, $P$ is a low discrepancy point set, and the error bound takes the form

$$
\left|I_{s}(f)-Q_{N, s}(f ; P)\right| \leq D(P) V(f),
$$

where $D(P)$ is the generalized discrepancy and $V(f)$ is the generalized variation of $f(\mathbf{x})$ Hic98. A special case of the above inequality is the well-known KoksmaHlawka inequality, for which $D(P)$ is the traditional star-discrepancy and $V(f)$ is variation of $f(\mathbf{x})$ in the sense of Hardy and Krause [Nie92]. The asymptotic convergence rate can be $O\left(N^{-1}(\log N)^{s}\right)$.

Dimensionally unbounded problems are frequently encountered in practice. For example, in particle and light transport problems and in simulations of stochastic processes, there is no a priori bound on the number of random numbers needed in one path simulation. The corresponding integral is of infinitely many variables. Many problems in mathematical finance can be expressed as Feynman-Kac formulas (see [Duf96]), and their solutions can be reduced to evaluations of infinite dimensional integrals. This paper considers integrals of functions of infinitely many variables. The corresponding problems are often called path integrations or functional integrations [WW96, TW98.

The infinite dimensional integral takes the form

$$
I_{\infty}(f)=\int_{C^{\infty}} f(\mathbf{x}) d \mathbf{x}
$$

where

$$
C^{\infty}:=[0,1]^{\infty}=\left\{\mathrm{x}=\left(x_{1}, x_{2}, \ldots\right): 0 \leq x_{i} \leq 1, i=1,2, \ldots\right\}
$$

is the infinite dimensional unit cube, and $d \mathbf{x}=d x_{1} d x_{2} \ldots$ is the infinite product of Lebesgue measures. For example, the measure of the interval $\left\{a_{n} \leq x_{n} \leq\right.$ $\left.b_{n}, n=1,2, \ldots\right\}$ equals $\prod_{n=1}^{\infty}\left(b_{n}-a_{n}\right)$ (see [Sob69, Sob98]). For a finite dimensional function, depending only on the variables $x_{1}, \cdots, x_{s}$, the integral (1) reduces to the common $s$-dimensional integral $I_{s}(f)$.

We are interested in QMC algorithms of the form

$$
Q_{N, \infty}(f)=Q_{N, \infty}(f ; P)=\frac{1}{N} \sum_{i=1}^{N} f\left(\mathbf{z}_{i}\right),
$$

where $P=\left\{\mathbf{z}_{1}, \ldots, \mathbf{z}_{N}\right\}$ is the set of $N$ points in the infinite dimensional unit cube $C^{\infty}$. The weights of the $\mathrm{QMC}$ algorithms are equal to $1 / N$. It is natural to ask under what conditions on the integrands and on the points does this quadrature rule approximate the integral well. Specifically, one would like to have a bound on the quadrature error. It would also be interesting to know under what conditions infinite dimensional integration has roughly the same difficulty as one dimensional integration. This is related to the question of tractability.

The concept of QMC tractability in infinite dimension is analogous to the finite dimensional case NW00, SW98. Let $\mathcal{H}$ be a normed space of functions defined on $C^{\infty}$, and let the norm in $\mathcal{H}$ be denoted by $\|\cdot\|_{\mathcal{H}}$. Let $P$ be a set of $N$ points in $C^{\infty}$. (Points in $P$ may be repeated.) Define the worst-case error of the algorithm 
$Q_{N, \infty}(\cdot ; P)$ by its worst-case performance over the unit ball in $\mathcal{H}$ :

$$
\operatorname{Err}\left(Q_{N, \infty}, \mathcal{H}\right)=\sup \left\{\left|I_{\infty}(f)-Q_{N, \infty}(f)\right|: f \in \mathcal{H},\|f\|_{\mathcal{H}} \leq 1\right\} .
$$

For a given sample size $N$, one would like to find the algorithm error for the best possible sample points $P$, that is,

$$
\begin{aligned}
\operatorname{Err}(N, \mathcal{H})=\inf _{P} \operatorname{Err}\left(Q_{N, \infty}, \mathcal{H}\right) & \\
& =\inf _{P} \sup \left\{\left|I_{\infty}(f)-Q_{N, \infty}(f)\right|: f \in \mathcal{H},\|f\|_{\mathcal{H}} \leq 1\right\} .
\end{aligned}
$$

For $N=0$, we formally set $Q_{0, \infty}(f)=0$, so the initial algorithm error is given as the norm of the functional $I_{\infty}(f)$ :

$$
\operatorname{Err}(0, \mathcal{H})=\sup \left\{\left|I_{\infty}(f)\right|: f \in \mathcal{H},\|f\|_{\mathcal{H}} \leq 1\right\}=\left\|I_{\infty}\right\| .
$$

One might ask what is the smallest $N$, for which there exists an algorithm $Q_{N, \infty}$, such that the initial error is reduced by a factor $\varepsilon$, where $\varepsilon \in(0,1]$, i.e., what is

$$
N(\varepsilon, \mathcal{H})=\min \{N: \operatorname{Err}(N, \mathcal{H}) \leq \varepsilon \operatorname{Err}(0, \mathcal{H})\} ?
$$

The problem of infinite dimensional integration, $I_{\infty}(f)$, is $Q M C$ tractable if there exist nonnegative constants $C$ and $p$ such that

$$
N(\varepsilon, \mathcal{H}) \leq C \varepsilon^{-p} \quad \forall \varepsilon \in(0,1] .
$$

The infimum of $p$ for which (5) holds is called the $\varepsilon$-exponent of QMC tractability.

A simple example of intractability in infinite dimension is due to Chentzov (see Sob69, Sob98]). Consider the set of functions

$$
\mathcal{F}=\left\{\left(x_{j}-x_{m}\right)^{2}: j, m=1,2, \ldots\right\},
$$

and suppose that the class $\mathcal{H}$ contains $\mathcal{F}$. For an arbitrary sequence $\left\{\mathbf{z}_{i}\right\}_{i=1}^{\infty}$ of points in $C^{\infty}$, and for any $N$, there exists a function $f \in \mathcal{F}$ such that

$$
\left|I_{\infty}(f)-Q_{N, \infty}\left(f ;\left\{\mathbf{z}_{i}\right\}_{i=1}^{N}\right)\right| \geq \frac{1}{12} .
$$

Thus, if $c=\sup _{f \in \mathcal{F}}\|f\|_{\mathcal{H}}$ is finite, it follows that $\operatorname{Err}\left(Q_{N, \infty}, \mathcal{H}\right) \geq(12 c)^{-1}$, and $N(\varepsilon, \mathcal{H})=\infty$ for all $\varepsilon<[12 c \operatorname{Err}(0, \mathcal{H})]^{-1}$. Therefore, the integration problem for this function class $\mathcal{H}$ is not QMC tractable.

In the example above all the coordinate directions have equal importance. However, the tractability situation may change completely if the dependence of $f(\mathbf{x})$ on successive variables is increasingly limited, i.e., the dependence of $f(\mathbf{x})$ on $x_{j}$ becomes weaker as $j$ increases. Such situations often occur in practice. For example, in the simulation of the trajectories of particles, successive collisions become less influential to the quantity in which we are interested. In mathematical finance, the payoff of a certain derivative security is less influenced by the interest rate of the time periods close to the expiration time. Sobol' Sob69, Sob98] studied some functions satisfying conditions of this type. In the finite dimensional case, Sloan and Woźniakowski [SW98] studied the strong tractability and tractability of weighted classes of functions.

This paper investigates the error bounds and tractability of certain weighted classes of functions as in [SW98], but in infinite dimension. Section 2 defines the relevant Hilbert spaces of integrands with very general reproducing kernels. It is shown that such Hilbert spaces can be decomposed as the direct sum of a series of their subspaces, each subspace containing functions of only a finite number of 
variables. In Section 3, the theory of reproducing kernel Hilbert spaces is used to derive a quadrature error bound, which takes the form of a product of a generalized discrepancy and a generalized variation. The general theory is illustrated by two concrete kernels, which correspond to the star-discrepancy and the centereddiscrepancy respectively. In Section 4, we establish the tractability of QMC algorithms for weighted classes of infinite dimensional functions, and prove that the $\varepsilon$-exponent is less than 2 under a very natural assumption. Moreover, under a somewhat stronger assumption on the weights, it is shown that the $\varepsilon$-exponent is 1. The proof is constructive.

\section{The PROJECTION DECOMPOSITION OF FUNCTIONS IN INFINITE DIMENSION}

In finite dimension, the derivation of QMC integration error bounds and the study of tractability of QMC algorithms rely on a decomposition of integrands into low dimensional parts and the theory of reproducing kernel Hilbert spaces Hic98. A similar approach is adopted here for the infinite dimensional case. The decomposition of the integrand into finite dimensional parts is called a projection decomposition.

Let $1: \infty$ denote the infinite set $\{1,2, \cdots\}$ of coordinate indices, and let $1: s$ denote the set $\{1,2, \cdots, s\}$ of the coordinate indices of the first $s$ variables. For any $u \subset 1: \infty$, let $|u|$ denote its cardinality, and let $C^{u}=[0,1]^{u}$ denote the $|u|$ dimensional unit cube involving the coordinates in $u$. Furthermore, let $\mathbf{x}_{u}$ denote the vector containing the coordinates of $\mathbf{x}$ whose indices are in $u$. By $\left(\mathbf{x}_{u}, \mathbf{1}\right)$ we mean the vector in $C^{\infty}$, where all the components $x_{j}$ with $j \notin u$ are set equal to 1 .

In order to obtain good estimators for the integral (1), we must define appropriate spaces of integrands, such that the dependence of $f(\mathbf{x})$ on successive variables, $x_{j}$, or sets of variables, $\mathbf{x}_{u}$, becomes weaker with increasing $j$ or $|u|$. For any set $u \subset 1: \infty$ with finite cardinality the nonnegative weight $\gamma_{u}$ indicates the importance of the variables indexed by $j \in u$. This approach was introduced in SW98. For the purpose of standardization, we set $\gamma_{\emptyset}=1$. An important special case is

$$
\gamma_{u}:=\prod_{j \in u} \gamma_{j}, u \subset 1: \infty
$$

for some nonnegative $\gamma_{1}, \gamma_{2}, \ldots$, but most of the results that follow are proved for arbitrary choices of $\gamma_{u}$. These weights must satisfy a summability condition, given below.

Define a reproducing kernel of the form

$$
K_{\infty}(\mathbf{x}, \mathbf{y})=\sum_{0 \leq|u|<\infty} \gamma_{u} \prod_{j \in u} k\left(x_{j}, y_{j}\right)
$$

where $k(x, y)$ is a symmetric, real-valued positive definite function on $[0,1]^{2}$. In the remainder of this paper the one-dimensional kernel $k$ and the weights $\gamma_{u}$ are always assumed to satisfy the following condition.

Assumption 1. The Hilbert space of univariate functions $H(k)$, admitting the reproducing kernel $k(x, y)$, does not contain any nonzero constant, that is

$$
H(1) \cap H(k)=\{0\},
$$

where $H(1)$ is the Hilbert space with kernel 1. Moreover, it is assumed that the kernel is bounded over the unit square, integrable over the unit square, and integrable 
along the diagonal:

$$
\begin{gathered}
L:=\sup _{(x, y) \in[0,1]^{2}}|k(x, y)|<\infty, \\
m:=\int_{[0,1]^{2}} k(x, y) d x d y \leq L, \quad M:=\int_{[0,1]} k(x, x) d x \leq L .
\end{gathered}
$$

It is also assumed that

$$
h(x)=\int_{0}^{1} k(x, y) d y \in H(k) .
$$

The summability condition on the weights is

$$
\sum_{0 \leq|u|<\infty} \gamma_{u} L^{|u|}<\infty
$$

If the $\gamma_{u}$ take the form (7), then this is equivalent to

$$
\sum_{j=1}^{\infty} \gamma_{j}<\infty
$$

Note that in fact $m \leq M$ since $k(x, y)$ is positive definite (see [HW00]). It follows from condition (12) that the function $K_{\infty}(\mathbf{x}, \mathbf{y})$ given by formula (8) is well defined. Moreover, it is positive definite. From the theory of reproducing kernel Hilbert spaces [Aro50, Sai88, Wah90] there exists a uniquely determined Hilbert space, admitting the reproducing kernel $K_{\infty}(\mathbf{x}, \mathbf{y})$. We denote this Hilbert space by $H\left(K_{\infty}\right)$.

To characterize the space $H\left(K_{\infty}\right)$ consider first the reproducing kernel

$$
\tilde{R}_{u}\left(\mathbf{x}_{u}, \mathbf{y}_{u}\right)=\prod_{j \in u} k\left(x_{j}, y_{j}\right)
$$

defined for finite sets $u \subset 1: \infty$. Denote the associated Hilbert space as $H_{u}$, with inner product and induced norm $\langle\cdot, \cdot\rangle_{H_{u}}$ and $\|\cdot\|_{H_{u}}$, respectively. When $u=\emptyset$, we define $\tilde{R}_{\emptyset}=1$, and the corresponding Hilbert space is $H_{\emptyset}=H(1)=\{f$ : $f$ is a constant\}. Also, consider the reproducing kernel

$$
R_{u}\left(\mathbf{x}_{u}, \mathbf{y}_{u}\right)=\gamma_{u} \tilde{R}_{u}\left(\mathbf{x}_{u}, \mathbf{y}_{u}\right)=\gamma_{u} \prod_{j \in u} k\left(x_{j}, y_{j}\right) .
$$

Denote the associated Hilbert space as $W_{u}$, with inner product and induced norm $\langle\cdot, \cdot\rangle_{W_{u}}$ and $\|\cdot\|_{W_{u}}$, respectively. The Hilbert space $H_{u}$ is closely related to the Hilbert space $W_{u}$. In fact, if $\gamma_{u}>0$, then $W_{u}=H_{u}$, and their inner products are related by

$$
\langle f, g\rangle_{W_{u}}=\gamma_{u}^{-1}\langle f, g\rangle_{H_{u}} .
$$

If $\gamma_{u}=0$, then $W_{u}=\{0\}$.

Lemma 2. Under Assumption 1 it follows that $H_{u} \cap H_{v}=\{0\}$ and $W_{u} \cap W_{v}=\{0\}$ for all $u \neq v$ where $u, v \subset 1: \infty$ and $|u|,|v|<\infty$.

Proof. Let $f$ be any function in $H_{u} \cap H_{v}$, and without loss of generality suppose that $\ell \in u-v$. Since $f \in H_{u}$, it follows that $f$ can be written as an infinite series 
in terms of the reproducing kernel $\tilde{R}_{u}$ :

$$
\begin{aligned}
f\left(\mathbf{x}_{u}\right) & =\sum_{i} c_{i} \tilde{R}_{u}\left(\mathbf{x}_{u}, \mathbf{y}_{i u}\right)=\sum_{i} c_{i} \prod_{j \in u} k\left(x_{j}, y_{i j}\right) \\
& =\sum_{i}\left[c_{i} \prod_{j \in u-\{\ell\}} k\left(x_{j}, y_{i j}\right)\right] k\left(x_{\ell}, y_{i \ell}\right) .
\end{aligned}
$$

For any fixed $\mathbf{x}_{u-\{\ell\}}$ the above series represents some function of $g\left(x_{\ell}\right) \in H(k)$. On the other hand, since $f \in H_{v}$ and $\ell \notin v$, the function $g\left(x_{\ell}\right)$ must be constant. By Assumption 1 it follows that $g\left(x_{\ell}\right)=0$. Since this holds no matter how $\mathbf{x}_{u-\{\ell\}}$ is fixed, the function $f$ must be zero.

Now consider the $s$-dimensional reproducing kernel $K_{s}\left(\mathbf{x}_{1: s}, \mathbf{y}_{1: s}\right)$ defined as

$$
K_{s}\left(\mathbf{x}_{1: s}, \mathbf{y}_{1: s}\right)=\sum_{u \subseteq 1: s} R_{u}\left(\mathbf{x}_{u}, \mathbf{y}_{u}\right)
$$

It is shown in the lemma below that the Hilbert space $H\left(K_{s}\right)$, admitting this kernel is simply the direct sum of the appropriate $W_{u}$.

Lemma 3. The Hilbert space $H\left(K_{s}\right)$ with the reproducing kernel $K_{s}\left(\mathbf{x}_{1: s}, \mathbf{y}_{1: s}\right)$ can be decomposed as the direct sum of Hilbert spaces $W_{u}$ with $u \subseteq 1: s$, that is

$$
H\left(K_{s}\right)=\bigoplus_{u \subseteq 1: s} W_{u}
$$

Any function $f\left(\mathbf{x}_{1: s}\right) \in H\left(K_{s}\right)$ has a unique decomposition

$$
f\left(\mathbf{x}_{1: s}\right)=\sum_{u \subseteq 1: s} f_{u}, \quad f_{u} \in W_{u}
$$

This is called the projection decomposition. The inner product and norm for $H\left(K_{s}\right)$ are related to the inner products and norms of the spaces $W_{u}$ as follows:

$$
\langle f, g\rangle_{H\left(K_{s}\right)}=\sum_{u \subseteq 1: s}\left\langle f_{u}, g_{u}\right\rangle_{W_{u}}, \quad\|f\|_{H\left(K_{s}\right)}^{2}=\sum_{u \subseteq 1: s}\left\|f_{u}\right\|_{W_{u}}^{2} .
$$

Proof. Since it was shown in Lemma 2 that $W_{u} \cap W_{v}=\{0\}$ for all $u \neq v$, one may define the Hilbert space $\tilde{W}_{s}=\bigoplus_{u \subseteq 1: s} W_{u}$. Any $f \in \tilde{W}_{s}$ may be written uniquely as $f=\sum_{u \subseteq 1: s} f_{u}$ with $f_{u} \in W_{u}$. One can then define an inner product and norm associated with $\tilde{W}_{s}$ in terms of the inner products and norms associated with the $W_{u}$ :

$$
\langle f, g\rangle_{\tilde{W}_{s}}=\sum_{u \subseteq 1: s}\left\langle f_{u}, g_{u}\right\rangle_{W_{u}}, \quad\|f\|_{\tilde{W}_{s}}^{2}=\sum_{u \subseteq 1: s}\left\|f_{u}\right\|_{W_{u}}^{2} .
$$

For this definition of the inner product it is straightforward to show that the reproducing kernel for $\tilde{W}_{s}$ is $K_{s}$ as defined in (14). Since each reproducing kernel corresponds to a unique Hilbert space, $H\left(K_{s}\right)=\tilde{W}_{s}$.

Now we are ready to discuss the infinite dimensional case. Consider the kernel, defined in (8), which can be rewritten as

$$
K_{\infty}(\mathbf{x}, \mathbf{y})=\sum_{|u|<\infty} R_{u}\left(\mathbf{x}_{u}, \mathbf{y}_{u}\right)
$$


This is the limit of $K_{s}$ when $s \rightarrow \infty$. Note that there are no terms with $|u|=\infty$ in the sum of (17). Let

$$
\tilde{W}_{\infty}=\left\{\sum_{|u|<\infty} f_{u}: f_{u} \in W_{u}, \sum_{|u|<\infty}\left\|f_{u}\right\|_{W_{u}}^{2}<\infty\right\} .
$$

Since $W_{u} \cap W_{v}=\{0\}$ for all $u \neq v$, any $f \in \tilde{W}_{\infty}$ has a unique projection decomposition

$$
f(\mathbf{x})=\sum_{|u|<\infty} f_{u}, \quad f_{u} \in W_{u} .
$$

Define the following inner product and norm for this space:

$$
\langle f, g\rangle_{\tilde{W}_{\infty}}=\sum_{|u|<\infty}\left\langle f_{u}, g_{u}\right\rangle_{W_{u}}, \quad\|f\|_{\tilde{W}_{\infty}}^{2}=\sum_{|u|<\infty}\left\|f_{u}\right\|_{W_{u}}^{2} .
$$

The following lemma shows that this Hilbert space has reproducing kernel (17).

Lemma 4. The space $\tilde{W}_{\infty}$ with inner product and the square norm defined in (20) is a Hilbert space with reproducing kernel (17), i.e., $H\left(K_{\infty}\right)=\tilde{W}_{\infty}$.

Proof. The fact that $\tilde{W}_{\infty}$ as defined is a Hilbert space is obvious. It remains to be shown that $K_{\infty}(\mathbf{x}, \mathbf{y})$ is its reproducing kernel. For any fixed $\mathbf{y} \in C^{\infty}$ it must be shown that $K_{\infty}(\mathbf{x}, \mathbf{y}) \in \tilde{W}_{\infty}$. From (17) it follows that $K_{\infty}(\mathbf{x}, \mathbf{y})=$ $\sum_{|u|<\infty} R_{u}\left(\mathbf{x}_{u}, \mathbf{y}_{u}\right)$ and each $R_{u}\left(\cdot, \mathbf{y}_{u}\right) \in W_{u}$. Moreover,

$$
\begin{aligned}
\sum_{|u|<\infty}\left\|R_{u}\left(\cdot, \mathbf{y}_{u}\right)\right\|_{W_{u}}^{2} & =\sum_{|u|<\infty}\left\langle R_{u}\left(\cdot, \mathbf{y}_{u}\right), R_{u}\left(\cdot, \mathbf{y}_{u}\right)\right\rangle_{W_{u}} \\
& =\sum_{|u|<\infty} R_{u}\left(\mathbf{y}_{u}, \mathbf{y}_{u}\right)=K_{\infty}(\mathbf{y}, \mathbf{y})<\infty
\end{aligned}
$$

by summability condition (12). Therefore, $K_{\infty}(\mathbf{x}, \mathbf{y}) \in \tilde{W}_{\infty}$ for any fixed $\mathbf{y} \in C^{\infty}$. Next, for any $f=\sum_{|u|<\infty} f_{u} \in \tilde{W}_{\infty}$ it follows that

$$
\left\langle f, K_{\infty}(\cdot, \mathbf{y})\right\rangle_{\tilde{W}_{\infty}}=\sum_{|u|<\infty}\left\langle f_{u}, R_{u}\left(\cdot, \mathbf{y}_{u}\right)\right\rangle_{W_{u}}=\sum_{|u|<\infty} f_{u}\left(\mathbf{y}_{u}\right)=f(\mathbf{y}),
$$

so $K_{\infty}(\mathbf{x}, \mathbf{y})$ has the reproducing property. The relation $H\left(K_{\infty}\right)=\tilde{W}_{\infty}$ follows from the uniqueness of Hilbert spaces admitting the same reproducing kernel $K_{\infty}(\mathbf{x}, \mathbf{y})$.

Corollary 5. The space $H\left(K_{\infty}\right)$ may also be defined as

$$
H\left(K_{\infty}\right)=\left\{\sum_{\substack{|u|<\infty \\ \gamma_{u}>0}} f_{u}: f_{u} \in H_{u}, \sum_{\substack{|u|<\infty \\ \gamma_{u}>0}} \gamma_{u}^{-1}\left\|f_{u}\right\|_{H_{u}}^{2}<\infty\right\} .
$$

If $s<d<\infty$, then $H\left(K_{s}\right)$ is a subspace of $H\left(K_{d}\right)$, which is a subspace of $H\left(K_{\infty}\right)$. The inner product for the Hilbert space $H\left(K_{s}\right)$ is the same as for $H\left(K_{d}\right)$ and $H\left(K_{\infty}\right)$ but restricted to the space of $s$-dimensional functions $H\left(K_{s}\right)$. 


\section{The error bounds of QuAsi-Monte Carlo Algorithms IN INFINITE DIMENSION}

Having defined an appropriate space of integrands we now derive the error bounds for quasi-Monte Carlo integration (2) using the theory of reproducing kernel Hilbert spaces. First, some notation is defined. Let $\|\cdot\|_{p}$ denote the $L^{p}$-norm of a function on $C^{u}$ with $|u|<\infty$, that is

$$
\|f\|_{p}=\left[\int_{C^{u}}|f|^{p} d \mathbf{x}_{u}\right]^{1 / p}, \quad\|f\|_{\infty}=\inf \{\lambda: f \leq \lambda \text { almost everywhere }\} .
$$

This notation is extended to the case of a series of projection terms $\left(f_{u}\right)$ where the range of $u$ is often $0 \leq|u|<\infty$ or $0<|u|<\infty$. If $f_{u}$ is a function on $C^{u}$ let

$$
\left\|\left(f_{u}\right)\right\|_{p}=\left[\sum_{u} \int_{C^{u}}\left|f_{u}\right|^{p} d \mathbf{x}_{u}\right]^{1 / p}, \quad\left\|\left(f_{u}\right)\right\|_{\infty}=\max _{u}\left\|f_{u}\right\|_{\infty} .
$$

3.1. General case. Consider now the infinite dimensional integration functional given by (11). Assumption (11) on $h(x)$ implies that one-dimensional integration is well defined for the space $H(k)$, and in fact $h(x)$ is the representer of the integration functional. Note that

$$
\|h\|_{H(k)}^{2}=\langle h, h\rangle_{H(k)}=\int_{0}^{1} h(x) d x=\int_{[0,1]^{2}} k(x, y) d x d y=m .
$$

It is straightforward to show that the function $\gamma_{u} \prod_{j \in u} h\left(x_{j}\right)$ is the representer of the integration functional for the Hilbert space $W_{u}$, and

$$
\left\|\gamma_{u} \prod_{j \in u} h\left(x_{j}\right)\right\|_{W_{u}}^{2}=\gamma_{u} m^{|u|} .
$$

Define the function

$$
h_{\infty}(\mathbf{x})=\int_{C^{\infty}} K_{\infty}(\mathbf{x}, \mathbf{y}) d \mathbf{y}=\sum_{|u|<\infty} \gamma_{u} \prod_{j \in u} h\left(x_{j}\right) .
$$

From the condition (12) it follows that $\left\|h_{\infty}\right\|_{H\left(K_{\infty}\right)}^{2}=\sum_{|u|<\infty} \gamma_{u} m^{|u|}<\infty$, so $h_{\infty} \in H\left(K_{\infty}\right)$. Integration on $C^{\infty}$ is a continuous, linear functional with representer $h_{\infty}$, i.e.,

$$
I_{\infty}(f)=\left\langle f, h_{\infty}\right\rangle_{H\left(K_{\infty}\right)} \quad \text { with } \quad h_{\infty}(\mathbf{y})=\int_{C^{\infty}} K_{\infty}(\mathbf{x}, \mathbf{y}) d \mathbf{x}
$$

The square norm of this functional is

$$
\left\|I_{\infty}\right\|^{2}=\left\|h_{\infty}\right\|_{H\left(K_{\infty}\right)}^{2}=\sum_{|u|<\infty} \gamma_{u} m^{|u|} .
$$

Because $H\left(K_{\infty}\right)$ has a reproducing kernel, $Q_{N, \infty}$ is a continuous, linear functional, and so is the integration error $I_{\infty}-Q_{N, \infty}$. By the Riesz Representation Theorem the quadrature error may be expressed as

$$
\left(I_{\infty}-Q_{N, \infty}\right)(f)=\langle\xi, f\rangle_{H\left(K_{\infty}\right)}, \forall f \in H\left(K_{\infty}\right)
$$

where the representer of the error functional is

$$
\xi(\mathbf{x})=\left\langle\xi, K_{\infty}(\cdot, \mathbf{x})\right\rangle_{H\left(K_{\infty}\right)}=\left(I_{\infty}-Q_{N, \infty}\right)\left(K_{\infty}(\cdot, \mathbf{x})\right) .
$$


Since QMC rules are exact for constants, the integration error for $f$ is the same as that for $f_{\perp}:=f-f_{\emptyset}$, the nonconstant part of the integrand $f$. The Cauchy-Schwarz inequality implies the following error bound

$$
\left|I_{\infty}(f)-Q_{N, \infty}(f)\right|=\left|\langle\xi, f\rangle_{H\left(K_{\infty}\right)}\right|=\left|\left\langle\xi, f_{\perp}\right\rangle_{H\left(K_{\infty}\right)}\right| \leq\|\xi\|_{H\left(K_{\infty}\right)}\left\|f_{\perp}\right\|_{H\left(K_{\infty}\right)} .
$$

This error bound is tight, since equality holds if $f(\mathbf{x})$ is a multiple of $\xi(\mathbf{x})$, the worst-case integrand.

The terms $\xi_{u}$ making up the projection decomposition of the worst-case integrand, $\xi(\mathbf{x})$, are the errors in integrating $R_{u}\left(\cdot, \mathbf{x}_{u}\right)$. Indeed,

$$
\begin{aligned}
\xi(\mathbf{x}) & =\left(I_{\infty}-Q_{N, \infty}\right)\left(K_{\infty}(\cdot, \mathbf{x})\right)=\left(I_{\infty}-Q_{N, \infty}\right) \sum_{0 \leq|u|<\infty} R_{u}\left(\cdot, \mathbf{x}_{u}\right) \\
& =\sum_{0 \leq|u|<\infty}\left(I_{\infty}-Q_{N, \infty}\right) R_{u}\left(\cdot, \mathbf{x}_{u}\right) .
\end{aligned}
$$

Note that $\left(I_{\infty}-Q_{N, \infty}\right) R_{u}\left(\cdot, \mathbf{x}_{u}\right) \in W_{u}$. According to the uniqueness of decomposition of functions in $H\left(K_{\infty}\right)$, we have $\xi_{\emptyset}=0$, and for $u \neq \emptyset$

$$
\xi_{u}(\mathbf{x})=\left(I_{\infty}-Q_{N, \infty}\right) R_{u}\left(\cdot, \mathbf{x}_{u}\right)=\gamma_{u} \tilde{\xi}_{u}(\mathbf{x})
$$

where

$$
\tilde{\xi}_{u}(\mathbf{x})=\left[\prod_{j \in u} \int_{0}^{1} k\left(z, x_{j}\right) d z-\frac{1}{N} \sum_{\mathbf{z} \in P} \prod_{j \in u} k\left(z_{j}, x_{j}\right)\right] .
$$

To show how each projection term $f_{u}$ in the series for the integrand $f$ contributes to the total integration error, the inner product $\left\langle\xi, f_{\perp}\right\rangle_{H\left(K_{\infty}\right)}$ is expressed in terms of $\left\langle\xi_{u}, f_{u}\right\rangle_{W_{u}}$ using Lemma 4:

$$
\begin{aligned}
\left|I_{\infty}(f)-Q_{N, \infty}(f)\right| & =\left|\langle\xi, f\rangle_{H\left(K_{\infty}\right)}\right|=\left|\left\langle\xi, f_{\perp}\right\rangle_{H\left(K_{\infty}\right)}\right| \\
& =\left|\sum_{\substack{0<|u|<\infty\\
}}\left\langle\xi_{u}, f_{u}\right\rangle_{W_{u}}\right|=\left|\sum_{\substack{0<|u|<\infty \\
\gamma_{u} \neq 0}}\left\langle\tilde{\xi}_{u}, f_{u}\right\rangle_{H_{u}}\right| \\
& \leq \sum_{\substack{0<|u|<\infty \\
\gamma_{u} \neq 0}} D_{2, u}(P) V_{2, u}(f) \\
& \leq D_{2}\left(P, K_{\infty}\right) V_{2}\left(f, K_{\infty}\right),
\end{aligned}
$$

where

$$
D_{2, u}(P)=\left\|\tilde{\xi}_{u}\right\|_{H_{u}}, \quad V_{2, u}(f)=\left\|f_{u}\right\|_{H_{u}}
$$


and

$$
\begin{gathered}
D_{2}\left(P, K_{\infty}\right)=\left\{\sum_{0<|u|<\infty} \gamma_{u} D_{2, u}^{2}(P)\right\}^{1 / 2}=\left\{\sum_{\substack{0<|u|<\infty \\
\gamma_{u} \neq 0}} \gamma_{u}\left\|\tilde{\xi}_{u}\right\|_{H_{u}}^{2}\right\}^{1 / 2} \\
=\left\{\sum_{\substack{0<|u|<\infty \\
\gamma_{u} \neq 0}} \gamma_{u}^{-1}\left\|\xi_{u}\right\|_{H_{u}}^{2}\right\}^{1 / 2}=|| \xi \|_{H\left(K_{\infty}\right)}, \\
V_{2}\left(f, K_{\infty}\right)=\left\{\sum_{\substack{0<|u|<\infty \\
\gamma_{u} \neq 0}} \gamma_{u}^{-1} V_{2, u}^{2}(f)\right\}^{1 / 2}=\left\{\sum_{\substack{0<|u|<\infty \\
\gamma_{u} \neq 0}} \gamma_{u}^{-1}\left\|f_{u}\right\|_{H_{u}}^{2}\right\}^{1 / 2} \\
\end{gathered}
$$

Definition 6. For any point set $P$ in the infinite dimensional unit cube $C^{\infty}$, the $L^{2}$-discrepancy is defined by (25) and is the norm of the worst-case integrand $\xi(\mathbf{x})$. The $L^{2}$-variation of $f(\mathbf{x})$ is defined by (26) and is the norm of the nonconstant part of $f(\mathbf{x})$.

The discrepancy depends on the point set $P$, but not on the integrand, $f(\mathbf{x})$, while the variation depends on the integrand but not on the point set. Both the discrepancy and the variation depend on the choice of the one-dimensional kernel, $k$, and the weights, $\gamma_{u}$.

Note that in the above derivation, one at first writes the error as the inner product of the worst-case integrand, $\xi$, with $f_{\perp}$. The inner product is expanded as a sum of inner products over $u$ with $0<|u|<\infty$, and the absolute value is taken inside the sum. The Cauchy-Schwarz inequality is applied to each inner product in the sum. The $u$ th term in this sum is multiplied and divided by a factor $\gamma_{u}^{-1 / 2}$. Finally the Cauchy-Schwarz inequality is again applied to the sum to obtain the error bound. The term $\left|\left\langle\tilde{\xi}_{u}, f_{u}\right\rangle_{H_{u}}\right|$ is the integration error for the projection term $f_{u}$, and this does not depend on $\gamma_{u}$. This error is no larger than the product $D_{2, u}(P) V_{2, u}(f)$.

Because the reproducing kernel $K_{\infty}(\mathbf{x}, \mathbf{y})$ is given by (8)), the $L^{2}$-discrepancy may be written in terms of integrals and sums involving the reproducing kernel:

$$
\begin{aligned}
& \text { (27) } D_{2}\left(P, K_{\infty}\right)=\langle\xi, \xi\rangle_{H\left(K_{\infty}\right)}^{1 / 2}=\left(I_{\infty}(\xi)-Q_{N, \infty}(\xi)\right)^{1 / 2} \\
& =\left\{\int_{C^{\infty}} \int_{C^{\infty}} K_{\infty}(\mathbf{x}, \mathbf{y}) d \mathbf{x} d \mathbf{y}-\frac{2}{N} \sum_{\mathbf{z} \in P} \int_{C^{\infty}} K_{\infty}(\mathbf{z}, \mathbf{y}) d \mathbf{y}+\frac{1}{N^{2}} \sum_{\mathbf{z}, \mathbf{z}^{\prime} \in P} K_{\infty}\left(\mathbf{z}, \mathbf{z}^{\prime}\right)\right\}^{1 / 2} .
\end{aligned}
$$

The following theorem summarizes these results.

Theorem 7. For any function $f(\mathbf{x}) \in H\left(K_{\infty}\right)$, the infinite dimensional QMC integration algorithm (2) has an error bound

$$
\left|I_{\infty}(f)-Q_{N, \infty}(f)\right|=\left|\langle\xi, f\rangle_{H\left(K_{\infty}\right)}\right| \leq D_{2}\left(P, K_{\infty}\right) V_{2}\left(f, K_{\infty}\right),
$$


where $D_{2}\left(P, K_{\infty}\right)$ and $V_{2}\left(f, K_{\infty}\right)$ are $L^{2}$-discrepancy and $L^{2}$-variation, respectively. Equality holds when $f(\mathbf{x})$ is a multiple of the worst-case integrand $\xi(\mathbf{x})$ as given in (22). The worst-case error of QMC algorithm $Q_{N, \infty}$ over the unit ball of $H\left(K_{\infty}\right)$ is the $L^{2}$-discrepancy:

$$
\operatorname{Err}\left(Q_{N, \infty}, H\left(K_{\infty}\right)\right)=D_{2}\left(P, K_{\infty}\right)
$$

Remark. If $f(\mathbf{x})$ is an $s$-dimensional function where $s$ is finite, then the sums over $u$ in formulas (25) and (26) need only be taken over $u \subseteq 1: s$. In this way we recover the error bounds of QMC algorithms for finite dimensional functions, which were studied by Hickernell [Hic98].

3.2. Star-discrepancy. Consider the following particular choice of kernel

$$
K_{\infty}^{*}(\mathbf{x}, \mathbf{y})=\sum_{|u|<\infty} \gamma_{u} \prod_{j \in u} k^{*}\left(x_{j}, y_{j}\right),
$$

where $k^{*}(x, y)=\min (1-x, 1-y)$. It is easy to check that $k^{*}(x, y)$ satisfies all the conditions in Assumption 1, Let $H_{u}^{*}$ denote the Hilbert space with the kernel $\prod_{j \in u} k^{*}\left(x_{j}, y_{j}\right)$. The inner product and the square norm are given by

$$
\left\langle f_{u}, g_{u}\right\rangle_{H_{u}^{*}}=\int_{C^{u}} \frac{\partial^{|u|} g_{u}}{\partial \mathbf{x}_{u}} \frac{\partial^{|u|} f_{u}}{\partial \mathbf{x}_{u}} d \mathbf{x}_{u}, \quad\left\|f_{u}\right\|_{H_{u}^{*}}^{2}=\int_{C^{u}}\left(\frac{\partial^{|u|} f_{u}}{\partial \mathbf{x}_{u}}\right)^{2} d \mathbf{x}_{u}
$$

for $f_{u}, g_{u} \in H_{u}^{*}$.

The projection term of the worst-case integrand $\xi(\mathbf{x})$ can be computed using (23) for $0<|u|<\infty$

$$
\xi_{u}(\mathbf{x})=\gamma_{u} \tilde{\xi}_{u}(\mathbf{x}), \quad \tilde{\xi}_{u}(\mathbf{x})=\left[\prod_{j \in u}\left(\frac{1}{2}-\frac{1}{2} x_{j}^{2}\right)-\frac{1}{N} \sum_{\mathbf{z} \in P} \prod_{j \in u}\left(1-\max \left(x_{j}, z_{j}\right)\right)\right] .
$$

Therefore,

$$
\begin{gathered}
\frac{\partial^{|u|} \tilde{\xi}_{u}}{\partial \mathbf{x}_{u}}=\left[\prod_{j \in u}\left(-x_{j}\right)-\frac{1}{N} \sum_{\mathbf{z} \in P} \prod_{j \in u}\left(-1_{\left\{z_{j}<x_{j}\right\}}\right)\right]=(-1)^{|u|} \operatorname{Disc}^{*}\left(u, \mathbf{x}_{u} ; P_{u}\right), \\
D_{2, u}^{*}(P)=\left\|\tilde{\xi}_{u}\right\|_{H_{u}}=\left\{\int_{C^{u}}\left[\operatorname{Disc}^{*}\left(u, \mathbf{x}_{u} ; P_{u}\right)\right]^{2} d \mathbf{x}_{u}\right\}^{1 / 2},
\end{gathered}
$$

where

$$
\operatorname{Disc}^{*}\left(u, \mathbf{x}_{u} ; P_{u}\right)=\operatorname{Vol}\left(\left[0, \mathbf{x}_{u}\right)\right)-\frac{1}{N}\left|P_{u} \cap\left[0, \mathbf{x}_{u}\right)\right|,
$$

and $P_{u}$ denotes the projection of the point set $P$ on the cube $C^{u}$, the notation $|A|$ means the number of points in the set $A$ counted with multiplicity. The geometric meaning of $\operatorname{Disc}^{*}\left(u, \mathbf{x}_{u} ; P_{u}\right)$ is the absolute difference between the volume of a box $\left[0, \mathbf{x}_{u}\right)$ and the proportion of points in $P_{u}$ that are also in that box (see [Hic98]). In this case the corresponding discrepancy is called the star-discrepancy. From the 
definition of discrepancy (25), the $L^{2}$-star-discrepancy takes the form

$$
\begin{aligned}
D_{2}^{*}(P)= & \left\{\sum_{0<|u|<\infty} \gamma_{u}\left[D_{2, u}^{*}(P)\right]^{2}\right\}^{1 / 2} \\
= & \left\{\sum_{0<|u|<\infty} \gamma_{u} \int_{C^{u}}\left(\operatorname{Disc}^{*}\left(u, \mathbf{x}_{u} ; P_{u}\right)\right)^{2} d \mathbf{x}_{u}\right\}^{1 / 2} \\
= & \left\{\sum _ { 0 < | u | < \infty } \gamma _ { u } \left[\left(\frac{1}{3}\right)^{|u|}-\frac{2}{N} \sum_{\mathbf{z} \in P} \prod_{j \in u}\left(\frac{1-z_{j}^{2}}{2}\right)\right.\right. \\
& \left.\left.+\frac{1}{N^{2}} \sum_{\mathbf{z}, \mathbf{z}^{\prime} \in P} \prod_{j \in u} \min \left(1-z_{j}, 1-z_{j}^{\prime}\right)\right]\right\}^{1 / 2} .
\end{aligned}
$$

Furthermore, from (27) the $L^{2}$-star-discrepancy of $P$ can be reduced to a double sum when the $\gamma_{u}$ satisfy (17):

$$
\begin{aligned}
{\left[D_{2}^{*}(P)\right]^{2}=} & \prod_{j=1}^{\infty}\left(1+\frac{\gamma_{j}}{3}\right)-\frac{2}{N} \sum_{\mathbf{z} \in P} \prod_{j=1}^{\infty}\left(1+\frac{\gamma_{j}}{2}\left(1-z_{j}^{2}\right)\right) \\
& +\frac{1}{N^{2}} \sum_{\mathbf{z}, \mathbf{z}^{\prime} \in P} \prod_{j=1}^{\infty}\left[1+\gamma_{j} \min \left(1-z_{j}, 1-z_{j}^{\prime}\right)\right] .
\end{aligned}
$$

The above formula is the limiting discrepancy, defined by Sloan and Woźniakowski SW98. It is clear that $D_{2}^{*}(P)$ is finite if and only if $\sum_{j=1}^{\infty} \gamma_{j}<\infty$, as noted in SW98, but obtained in a somewhat different manner.

Now consider the variation of the integrand $f(\mathbf{x})$. For a fixed subset $u \subset 1: \infty$ with $0<|u|<\infty$ recall that the reproducing kernel for $H_{u}^{*}$ is $\prod_{j \in u}\left[1-\max \left(x_{j}, y_{j}\right)\right]$. Thus, for any $f_{u} \in H_{u}^{*}$, it follows that $f\left(\mathbf{x}_{u}\right)=0$ when $x_{j}=1$ for any $j \in u$. Thus, if $f=\sum_{|u|<\infty} f_{u}$ is the projection decomposition of any $f \in H\left(K_{\infty}^{*}\right)$, then

$$
f\left(\mathbf{x}_{u}, \mathbf{1}\right)=\sum_{v \subseteq u} f_{v}\left(\mathbf{x}_{v}\right), \quad \frac{\partial^{|u|}}{\partial \mathbf{x}_{u}} f\left(\mathbf{x}_{u}, \mathbf{1}\right)=\frac{\partial^{|u|} f_{u}}{\partial \mathbf{x}_{u}}
$$

Therefore, from (26) the $L^{2}$-variation can be written as

$$
V_{2}^{*}(f)=\left\|\left(\gamma_{u}^{-\frac{1}{2}} \frac{\partial^{|u|}}{\partial \mathbf{x}_{u}} f\left(\mathbf{x}_{u}, \mathbf{1}\right)\right)_{\substack{0<|u|<\infty \\ \gamma_{u} \neq 0}}\right\|_{2} .
$$

What kind of function belongs to $H\left(K_{\infty}^{*}\right)$ ? As an example, suppose that $\phi(\mathbf{x})$ is a function of infinitely many variables with all partial derivatives $\partial^{|u|} \phi_{u} / \partial \mathbf{x}_{u}$ continuous and uniformly bounded. Then the function $f(\mathbf{x})=\phi\left(\gamma_{1} x_{1}, \cdots, \gamma_{j} x_{j}, \cdots\right)$ belongs to $H\left(K_{\infty}^{*}\right)$ assuming that the $\gamma_{u}$ are defined by (7) and that the $\gamma_{j}$ satisfy Assumption 1 Indeed,

$$
\frac{\partial^{|u|} f_{u}}{\partial \mathbf{x}_{u}}=\gamma_{u} \frac{\partial^{|u|} \phi_{u}}{\partial \mathbf{x}_{u}}
$$

so it is easy to see that $\|f\|_{H\left(K_{\infty}^{*}\right)}$ is finite. Here we can also see the role of the weights $\gamma_{j}$. 
Since in this special case the inner product of $H_{u}^{*}$ takes the form (29), one can also define the so-called $L^{p}$-star-discrepancy and $L^{q}$-variation in terms of the $L^{p_{-}}$ norm. Following similar lines as in (24) and applying the Hölder inequality instead of Cauchy-Schwarz inequality, we obtain the following family of error bounds

$$
\left|I_{\infty}(f)-Q_{N, \infty}(f)\right| \leq D_{p}^{*}(P) V_{q}^{*}(f), \quad p^{-1}+q^{-1}=1, p \in[1, \infty]
$$

where

$$
D_{p}^{*}(P)=\left\|\left(\gamma_{u}^{\frac{1}{2}} \frac{\partial^{|u|} \tilde{\xi}_{u}}{\partial \mathbf{x}_{u}}\right)_{\substack{0<|u|<\infty\\}}\right\|_{p}, \quad V_{q}^{*}(f)=\left\|\left(\gamma_{u}^{-\frac{1}{2}} \frac{\partial^{|u|} f_{u}}{\partial \mathbf{x}_{u}}\right)_{\substack{0<|u|<\infty \\ \gamma u \neq 0}}\right\|_{q} .
$$

The quantities $D_{p}^{*}(P)$ and $V_{q}^{*}(f)$ are called the $L^{p}$-star-discrepancy of the point set $P$ and the $L^{q}$-variation of $f(\mathbf{x})$, respectively. Using the formulas for $\partial^{|u|} \tilde{\xi}_{u} / \partial \mathbf{x}_{u}$ and $\partial^{|u|} f_{u} / \partial \mathbf{x}_{u}$, one obtains the formulas

$$
\begin{gathered}
D_{p}^{*}(P)=\left\|\left(\gamma_{u}^{\frac{1}{2}} \operatorname{Disc}^{*}\left(u, \mathbf{x}_{u} ; P_{u}\right)\right)_{\substack{0<|u|<\infty \\
V_{q}}}\right\|_{p}, \\
V^{*}(f)=\left\|\left(\gamma_{u}^{-\frac{1}{2}} \frac{\partial^{|u|}}{\partial \mathbf{x}_{u}} f\left(\mathbf{x}_{u}, \mathbf{1}\right)\right)_{\substack{0<|u|<\infty \\
\gamma_{u} \neq 0}}\right\|_{q} .
\end{gathered}
$$

For $p<\infty$, the $p^{\text {th }}$ power of the discrepancy, $\left[D_{p}^{*}(P)\right]^{p}$, is the weighted sum of the terms $\left\|\operatorname{Disc}^{*}\left(u, \mathbf{x}_{u} ; P_{u}\right)\right\|_{p}^{p}$, which are the $p^{\text {th }}$ powers of the traditional $L^{p}$-stardiscrepancy of the projections $P_{u}$ (see [MC94]) . For $p=\infty$,

$$
D_{\infty}^{*}(P)=\sup _{\mathbf{x} \in C^{\infty}} \max _{0<|u|<\infty} \gamma_{u}^{\frac{1}{2}} \operatorname{Disc}^{*}\left(u, \mathbf{x}_{u} ; P_{u}\right)
$$

3.3. Centered-discrepancy. Now consider another choice of kernel:

$$
K_{\infty}^{c}(\mathbf{x}, \mathbf{y})=\sum_{|u|<\infty} \gamma_{u} \prod_{j \in u} k^{c}\left(x_{j}, y_{j}\right)
$$

with

$$
k^{c}(x, y)=\frac{1}{2}|x-1 / 2|+\frac{1}{2}|y-1 / 2|-\frac{1}{2}|x-y| .
$$

It is also easy to check that $k^{c}(x, y)$ satisfies all the conditions in Assumption 1 Let $H_{u}^{c}$ denote the Hilbert space with the kernel $\prod_{j \in u} k^{c}\left(x_{j}, y_{j}\right)$. The inner product and the square norm are given by

$$
\left\langle f_{u}, g_{u}\right\rangle_{H_{u}^{c}}=\int_{C^{u}} \frac{\partial^{|u|} g_{u}}{\partial \mathbf{x}_{u}} \frac{\partial^{|u|} f_{u}}{\partial \mathbf{x}_{u}} d \mathbf{x}_{u}, \quad\left\|f_{u}\right\|_{H_{u}^{c}}^{2}=\int_{C^{u}}\left(\frac{\partial^{|u|} f_{u}}{\partial \mathbf{x}_{u}}\right)^{2} d \mathbf{x}_{u}
$$

for any $f_{u}, g_{u} \in H_{u}^{c}$. The projection term of the worst-case integrand can be computed by using (23) for $0<|u|<\infty$ :

$$
\begin{gathered}
\xi_{u}(\mathbf{x})=\gamma_{u} \tilde{\xi}_{u}(\mathbf{x}), \\
\tilde{\xi}_{u}(\mathbf{x})=\left[\prod_{j \in u}\left(\frac{1}{2}\left|x_{j}-\frac{1}{2}\right|-\frac{1}{2}\left|x_{j}-\frac{1}{2}\right|^{2}\right)-\frac{1}{N} \sum_{\mathbf{z} \in P} \prod_{j \in u} k^{c}\left(x_{j}, z_{j}\right)\right] .
\end{gathered}
$$


Therefore,

$$
\frac{\partial^{|u|} \tilde{\xi}_{u}}{\partial \mathbf{x}_{u}}=\operatorname{Disc}^{c}\left(u, \mathbf{x}_{u} ; P_{u}\right)
$$

where

$$
\operatorname{Disc}^{c}\left(u, \mathbf{x}_{u} ; P_{u}\right)=\prod_{j \in u}\left(-x_{j}+1_{\left\{x_{j}>1 / 2\right\}}\right)-\frac{1}{N} \sum_{\mathbf{z} \in P} \prod_{j \in u}\left(1_{\left\{x_{j}>1 / 2\right\}}-1_{\left\{x_{j}>z_{j}\right\}}\right) .
$$

For the geometric meaning of $\operatorname{Disc}^{c}\left(u, \mathbf{x}_{u} ; P_{u}\right)$ see Hickernell [Hic98]. In the same way as in the star-discrepancy case, one can obtain that for any $f(\mathbf{x}) \in H\left(K_{\infty}^{c}\right)$ and $0<|u|<\infty$,

$$
\frac{\partial^{|u|} f_{u}}{\partial \mathbf{x}_{u}}=\frac{\partial^{|u|}}{\partial \mathbf{x}_{u}} f\left(\mathbf{x}_{u}, \mathbf{0 . 5}\right)
$$

The error bounds can be derived similarly. The formulas for the $L^{p}$-centereddiscrepancy and the corresponding $L^{q}$-variation are

$$
\begin{aligned}
& D_{p}^{c}(P)=\left\|\left(\gamma_{u}^{\frac{1}{2}} \operatorname{Disc}^{c}\left(u, \mathbf{x}_{u} ; P_{u}\right)\right)_{\substack{0<|u|<\infty \\
V_{q}^{c}}}\right\|_{p}, \\
& V^{c}(f)=\left\|\left(\gamma_{u}^{-\frac{1}{2}} \frac{\partial^{|u|}}{\partial \mathbf{x}_{u}} f\left(\mathbf{x}_{u}, \mathbf{0 . 5}\right)\right)_{\substack{0<|u|<\infty \\
\gamma_{u} \neq 0}}\right\|_{q} .
\end{aligned}
$$

For $p=2$ and $\gamma_{u}$ of the form (77) the $L^{2}$-centered-discrepancy takes the form

$$
\begin{aligned}
{\left[D_{2}^{c}(P)\right]^{2}=} & \prod_{j=1}^{\infty}\left(1+\frac{\gamma_{i}}{12}\right)-\frac{2}{N} \sum_{\mathbf{z} \in P} \prod_{j=1}^{\infty}\left[1+\frac{\gamma_{j}}{2}\left(\left|z_{j}-\frac{1}{2}\right|-\left|z_{j}-\frac{1}{2}\right|^{2}\right)\right] \\
& +\frac{1}{N^{2}} \sum_{\mathbf{z}, \mathbf{z}^{\prime} \in P} \prod_{j=1}^{\infty}\left[1+\frac{\gamma_{j}}{2}\left(\left|z_{j}-\frac{1}{2}\right|+\left|z_{j}^{\prime}-\frac{1}{2}\right|-\left|z_{j}-z_{j}^{\prime}\right|\right)\right] .
\end{aligned}
$$

Clearly, $D_{2}^{c}(P)$ is finite if and only if $\sum_{j=1}^{\infty} \gamma_{j}<\infty$.

\section{Tractability of quasi-Monte Carlo algorithms IN INFINITE DIMENSION}

The concept of QMC tractability in infinite dimension was described in the introduction. An example of intractability is also given there. That example shows that we may have intractability of QMC algorithms even for a relatively simple class of functions, if all variables are equally important. It is natural to ask when are QMC algorithms tractable in infinite dimension, and when is the $\varepsilon$-exponent the same as for the one-dimensional case?

Let $B\left(K_{\infty}\right)$ denote the unit ball in the space $H\left(K_{\infty}\right)$, i.e.,

$$
B\left(K_{\infty}\right)=\left\{f \in H\left(K_{\infty}\right):\|f\|_{H\left(K_{\infty}\right)} \leq 1\right\}
$$

Note that by (4) and Theorem 7 it follows that

$$
\operatorname{Err}\left(N, H\left(K_{\infty}\right)\right)=\inf _{P} \sup _{f \in B\left(K_{\infty}\right)}\left|I_{\infty}(f)-Q_{N, \infty}(f)\right|=\inf _{P} D_{2}\left(P, K_{\infty}\right) .
$$

Thus, the problem of tractability becomes one of determining how small the discrepancy can be made using $N$ sample points. 
4.1. When are QMC algorithms tractable? Consider the reproducing kernel $K_{\infty}(\mathbf{x}, \mathbf{y})$ defined in (8) and the corresponding weighted class of functions $H\left(K_{\infty}\right)$. We will show that for $H\left(K_{\infty}\right)$ the infinite dimensional integration problem is tractable.

Theorem 8. If Assumption 11 holds, then the infinite dimensional QMC integration algorithm (2) is QMC tractable for Hilbert space $H\left(K_{\infty}\right)$, and the $\varepsilon$-exponent satisfies $p^{*} \leq 2$.

Proof. The proof is obtained by looking at the performance of a simple Monte Carlo algorithm. The same technique was employed in [SW98] in the finite dimensional case.

Recall the formula for the discrepancy in terms of the reproducing kernel in (27). Assume that $P$ consists of independent points all uniformly distributed on $[0,1)^{\infty}$. Then take the expectation of the square discrepancy

$$
\begin{aligned}
& E_{P}\left[D_{2}\left(P, K_{\infty}\right)\right]^{2}=\int_{C^{\infty}} \int_{C^{\infty}} K_{\infty}(\mathbf{x}, \mathbf{y}) d \mathbf{x} d \mathbf{y}-2 \int_{C^{\infty}} \int_{C^{\infty}} K_{\infty}(\mathbf{x}, \mathbf{y}) d \mathbf{x} d \mathbf{y} \\
&+\frac{1}{N^{2}}\left(N \int_{C^{\infty}} K_{\infty}(\mathbf{x}, \mathbf{x}) d \mathbf{x}+\left(N^{2}-N\right) \int_{C^{\infty}} \int_{C^{\infty}} K_{\infty}(\mathbf{x}, \mathbf{y}) d \mathbf{x} d \mathbf{y}\right) \\
&=\frac{1}{N}\left(\int_{C^{\infty}} K_{\infty}(\mathbf{x}, \mathbf{x}) d \mathbf{x}-\int_{C^{\infty}} \int_{C^{\infty}} K_{\infty}(\mathbf{x}, \mathbf{y}) d \mathbf{x} d \mathbf{y}\right)
\end{aligned}
$$

This formula also appears in Hic98 and elsewhere.

Assumption 1 and (21) imply that the two integrals appearing above are finite, namely,

$$
\begin{gathered}
\int_{C^{\infty}} K_{\infty}(\mathbf{x}, \mathbf{x}) d \mathbf{x}=\sum_{|u|<\infty} \gamma_{u} \prod_{j \in u} \int_{0}^{1} k\left(x_{j}, x_{j}\right) d x_{j}=\sum_{|u|<\infty} \gamma_{u} M^{|u|}<\infty \\
\left\|I_{\infty}\right\|^{2}=\int_{C^{\infty}} K_{\infty}(\mathbf{x}, \mathbf{y}) d \mathbf{x} d \mathbf{y}=\sum_{|u|<\infty} \gamma_{u} m^{|u|}<\infty
\end{gathered}
$$

Under the assumption that $\gamma_{\emptyset}=1$, it follows that $\left\|I_{\infty}\right\| \geq 1$.

The best discrepancy possible is certainly no worse than the root mean square discrepancy produced by Monte Carlo quadrature. Combining formulas (32) and (33) together with the above equations now implies that

$$
\left[\frac{\operatorname{Err}\left(N, H\left(K_{\infty}\right)\right)}{\operatorname{Err}\left(0, H\left(K_{\infty}\right)\right)}\right]^{2} \leq \frac{E_{P}\left[D_{2}\left(P, K_{\infty}\right)\right]^{2}}{\left\|I_{\infty}\right\|^{2}} \leq \frac{1}{N} \sum_{|u|<\infty} \gamma_{u} M^{|u|}
$$

This implies that $\operatorname{Err}\left(N, H\left(K_{\infty}\right)\right) \leq \varepsilon \operatorname{Err}\left(0, H\left(K_{\infty}\right)\right)$ for

$$
N=\varepsilon^{-2} \sum_{|u|<\infty} \gamma_{u} M^{|u|} \text {. }
$$

So integration is tractable with the $\varepsilon$-exponent satisfying $p^{*} \leq 2$.

A lower bound on the $\varepsilon$-exponent may be found by considering how small one can make the discrepancy for one-dimensional integration, i.e., by setting $\gamma_{u}=0$ for all $u \neq \emptyset,\{1\}$. The answer depends on the smoothness of the reproducing kernel $k(x, y)$, or equivalently, the smoothness of the functions in $H(k)$. For the examples 
of the star- and centered-discrepancy given in the previous section, it is known that $p^{*} \geq 1$. The next subsection answers the question of when the $\varepsilon$-exponent equals one for the infinite dimensional case.

4.2. When is the $\varepsilon$-exponent of QMC tractability $\mathbf{1}$ for the star-discrepancy? This subsection considers the specific kernel $K_{\infty}^{*}(\mathbf{x}, \mathbf{y})$ defined by (28) and its corresponding Hilbert space $H\left(K_{\infty}^{*}\right)$. The corresponding discrepancy is the stardiscrepancy. Consider the unit ball in $H\left(K_{\infty}^{*}\right)$, that is, $B\left(K_{\infty}^{*}\right)=\left\{f \in H\left(K_{\infty}^{*}\right)\right.$ : $\left.\|f\|_{H\left(K_{\infty}^{*}\right)} \leq 1\right\}$. The notation $\operatorname{Disc}^{*}\left(u, \mathbf{x}_{u} ; P_{u}\right)$ retains the same meaning as in Section 3. Under a somewhat stronger assumption on the weights $\left\{\gamma_{j}\right\}$ than Assumption 1, it is shown that the $\varepsilon$-exponent of QMC tractability is 1. The proof is constructive.

The multidimensional Halton sequence is defined in Hal60. This definition is now extended to infinite dimension. Let $b_{j}$ be the $j$ th prime number. Define the infinite dimensional Halton sequence to be the sequence $\mathcal{S}=\left\{\mathbf{x}_{0}, \mathbf{x}_{1}, \cdots\right\}$ with

$$
\mathbf{x}_{n}=\left(\phi_{b_{1}}(n), \phi_{b_{2}}(n), \cdots, \phi_{b_{j}}(n), \cdots\right), n=0,1,2, \cdots,
$$

where $\left\{\phi_{b_{j}}(n)\right\}$ is the van der Corput sequence in base $b_{j}$. Let $u$ be a subset of $1: \infty$ with $|u|<\infty$. The projection of the infinite dimensional Halton sequence $\mathcal{S}$ on the cube $C^{u}$ is a $|u|$-dimensional Halton sequence (denoted by $\mathcal{S}_{u}$ ) in bases $b_{j}$ with $j \in u$.

Theorem 9. Assume that in the QMC algorithm (2), the points are taken to be the first $N$ points of an infinite dimensional Halton sequence. If the weights $\gamma_{u}$ satisfy

$$
\gamma_{u}=\prod_{j \in u} \gamma_{j}, \quad \sum_{j=1}^{\infty} \gamma_{j}^{1 / 2} j \log j<\infty,
$$

or if there exists some $s<\infty$ such that

$$
\gamma_{u}=\left\{\begin{array}{ll}
\prod_{j \in u} \gamma_{j}, & |u| \leq s, \\
0, & |u|>s,
\end{array} \quad \sum_{j=1}^{\infty} \gamma_{j} j^{2} \log ^{2} j<\infty,\right.
$$

then for any $\delta>0$, there exists a constant $C_{*}$, such that

$$
D_{2}^{*}\left(\mathcal{S}_{N}\right)=\sup _{f \in B\left(K_{\infty}^{*}\right)}\left|I_{\infty}(f)-Q_{N, \infty}(f)\right| \leq C_{*} N^{-1+\delta} .
$$

Consequently, the $\varepsilon$-exponent of QMC tractability equals 1 .

Proof. Recall from (30) that the $L^{2}$-star-discrepancy may be written as

$$
\begin{aligned}
D_{2}^{*}\left(\mathcal{S}_{N}\right) & =\left\{\sum_{0<|u|<\infty} \gamma_{u}\left[D_{2, u}^{*}\left(\mathcal{S}_{u N}\right)\right]^{2}\right\}^{1 / 2}, \\
D_{2, u}^{*}\left(\mathcal{S}_{u N}\right) & =\left\{\int_{C^{u}}\left[\operatorname{Disc}^{*}\left(u, \mathbf{x}_{u} ; \mathcal{S}_{u N}\right)\right]^{2} d \mathbf{x}_{u}\right\}^{1 / 2} .
\end{aligned}
$$

The traditional (extreme) star-discrepancy of the first $N$ points of the Halton sequence can be bounded (see [Nie92]), and this serves as an upper bound for the 
pieces making up the $L^{2}$-star-discrepancy:

$$
D_{2, u}^{*}\left(\mathcal{S}_{u N}\right) \leq \frac{|u|}{N}+\frac{1}{N} \prod_{j \in u} \alpha_{j}, \quad \text { for all } \quad N \geq 1
$$

where

$$
\alpha_{j}=\beta_{j} \log N+\frac{1}{2}\left(b_{j}+1\right), \quad \beta_{j}=\frac{b_{j}-1}{2 \log b_{j}} .
$$

It is well known that the $j$ th prime number $b_{j}$ is $O(j \log j)$ when $j \rightarrow \infty$, so $\beta_{j} \sim j$, and

$$
D_{2, u}^{*}\left(\mathcal{S}_{u N}\right) \leq \frac{1}{N} \prod_{j \in u}\left(\alpha_{j}+1\right) \leq \frac{1}{N} \prod_{j \in u}\left[C_{1} j \log (j+1) \log (e N)\right]
$$

for some constant $C_{1}>0$, where $e \approx 2.718$ is the antilogarithm of 1 . This leads to a bound on the square $L^{2}$-star-discrepancy of

$$
\left[D_{2}^{*}\left(\mathcal{S}_{N}\right)\right]^{2} \leq \frac{1}{N^{2}} \sum_{0<|u|<\infty} \gamma_{u} \prod_{j \in u}\left[C_{1} j \log (j+1) \log (e N)\right]^{2}
$$

If the $\gamma_{u}$ satisfy (34), then for any $\delta>0$ choose an $\ell$ such that

$$
\sum_{j=\ell+1}^{\infty} \gamma_{j}^{1 / 2} j \log (j+1) \leq \delta / C_{1}
$$

and define

$$
\begin{gathered}
C_{2}=\min \left(1, \delta C_{1}^{-1}\left\{\sum_{j=1}^{\ell} \gamma_{j}^{1 / 2} j \log (j+1)\right\}^{-1}\right), \\
w_{j}= \begin{cases}C_{2}^{2} \gamma_{j}, & j=1, \ldots, \ell, \\
\gamma_{j}, & j=\ell+1, \ell+2, \ldots\end{cases}
\end{gathered}
$$

Note from these definitions that $w_{u}=\prod_{j \in u} w_{j} \geq C_{2}^{2 \ell} \gamma_{u}$, and

$$
\sum_{j=1}^{\infty} w_{j}^{1 / 2} j \log (j+1)=C_{2} \sum_{j=1}^{\ell} \gamma_{j}^{1 / 2} j \log (j+1)+\sum_{j=\ell+1}^{\infty} \gamma_{j}^{1 / 2} j \log (j+1) \leq 2 \delta / C_{1} .
$$


Using (37), the binomial theorem, and some elementary properties of exponentials and logarithms one may then derive the following upper bound on the discrepancy:

$$
\begin{aligned}
{\left[D_{2}^{*}\left(\mathcal{S}_{N}\right)\right]^{2} } & \leq \frac{1}{N^{2} C_{2}^{2 \ell}} \sum_{0<|u|<\infty} w_{u} \prod_{j \in u}\left[C_{1} j \log (j+1) \log (e N)\right]^{2} \\
& \leq \frac{1}{N^{2} C_{2}^{2 \ell}} \prod_{j=1}^{\infty}\left\{1+w_{j}\left[C_{1} j \log (j+1) \log (e N)\right]^{2}\right\} \\
& \leq \frac{1}{N^{2} C_{2}^{2 \ell}} \exp \left(\sum_{j=1}^{\infty} \log \left\{1+w_{j}\left[C_{1} j \log (j+1) \log (e N)\right]^{2}\right\}\right) \\
& \leq \frac{1}{N^{2} C_{2}^{2 \ell}} \exp \left(\sum_{j=1}^{\infty} w_{j}^{1 / 2}\left[C_{1} j \log (j+1) \log (e N)\right]\right) \\
& =\frac{1}{N^{2} C_{2}^{2 \ell}} \exp \left(C_{1} \log (e N) \sum_{j=1}^{\infty} w_{j}^{1 / 2} j \log (j+1)\right) \\
& \leq \frac{1}{N^{2} C_{2}^{2 \ell}} \exp [2 \delta \log (e N)]=C_{*}^{2} N^{-2+2 \delta},
\end{aligned}
$$

where $C_{*}=e^{\delta} / C_{2}^{\ell}$. This completes the proof under condition (34).

When condition (35) is satisfied, note that this implies that

$$
\sum_{0<|u|<\infty} \gamma_{u} \prod_{j \in u}[j \log (j+1)]^{2} \leq \sum_{d=1}^{s}\left\{\sum_{j=1}^{\infty} \gamma_{j}[j \log (j+1)]^{2}\right\}^{d}<\infty
$$

Define

$$
C_{3}=\sum_{0<|u|<\infty}\left\{\gamma_{u} \prod_{j \in u}[j \log (j+1)]^{2}\right\} \max _{d=1, \ldots, s}\left(\frac{C_{1}}{2 \delta}\right)^{2 d}(2 d) ! .
$$

It now follows from (37) that

$$
\begin{aligned}
{\left[D_{2}^{*}\left(\mathcal{S}_{N}\right)\right]^{2} } & \leq \frac{1}{N^{2}} \sum_{d=1}^{s}\left(\left[C_{1} \log (e N)\right]^{2 d} \sum_{|u|=d}\left\{\gamma_{u} \prod_{j \in u}[j \log (j+1)]^{2}\right\}\right) \\
& \leq \frac{C_{3}}{N^{2}} \sum_{d=1}^{s} \frac{[2 \delta \log (e N)]^{2 d}}{(2 d) !} \leq \frac{C_{3}}{N^{2}} \exp [2 \delta \log (e N)]=C_{*}^{2} N^{-2+2 \delta},
\end{aligned}
$$

where $C_{*}=\sqrt{C_{3}} e^{\delta}$. This completes the proof under the hypothesis (35).

The proof of Theorem 9 is constructive. Some other low discrepancy sequences, for example, the Sobol' sequence [Sob69], some Niederreiter sequences [Nie92] and some Niederreiter-Xing sequences [NX96] also have infinite dimensional versions. The upper bounds for the traditional star-discrepancy of these sequences may be better than that of the Halton sequence. Thus, one may be able to construct an infinite dimensional QMC algorithm with $\varepsilon$-exponent equal to 1 under slightly weaker assumptions on the weights than those above. Note, however, that the Faure sequence Fau82 has no infinite dimensional version, since one must use a prime base no smaller than the dimension of the sequence. 


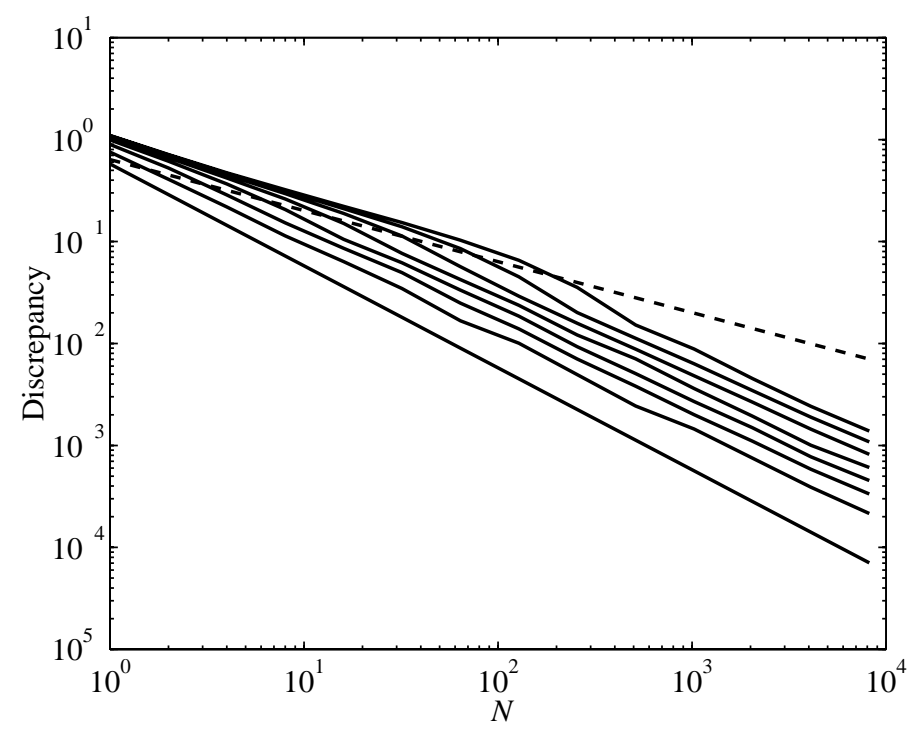

Figure 1. The $L^{2}$-star-discrepancy of the Halton sequence (solid) for the choice of weights (38a) for $s=1,2,4, \ldots, 128$ and $N=$ $1,2,4, \ldots, 8192$ and the root mean square $L^{2}$-star-discrepancy of a simple random sample (dashed) for the same choice of weights with $s=\infty$.

4.3. Computational investigation of infinite dimensional low discrepancy sequences. Theorem 7 shows that the discrepancy is the worst-case error of the QMC algorithm over the unit ball in $H\left(K_{\infty}\right)$. Theorems 8 and 9 give upper bounds on the $\varepsilon$-exponent under different conditions on the weights. It is interesting to investigate empirically the influence of the weights on the discrepancy of an actual infinite dimensional low discrepancy sequence. The traditional discrepancies in finite dimension were studied empirically in [MC94].

In practice, one cannot exactly compute the discrepancy of an infinite dimensional sequence. However, one may do the following. Suppose that the $\gamma_{u}$ satisfy (77), and the $\gamma_{j}$ satisfy one of two possibilities:

$$
\begin{array}{ll}
\gamma_{j}=j^{-2}, j=1,2, \ldots, s, & \gamma_{j}=0, j=s+1, s+2, \ldots, \\
\gamma_{j}=2^{-j}, j=1,2, \cdots, s, & \gamma_{j}=0, j=s+1, s+2, \ldots,
\end{array}
$$

for some fixed $s$. The $L^{2}$-star-discrepancy of the Halton sequence is computed for these two choices of weights for $s=1,2,4,8,16,32,64,128$. As $s$ increases the discrepancy approaches the infinite dimensional case. The root mean square $L^{2}$ star-discrepancy of a simple random sample is also shown for reference. These plots are shown in Figures 1 and 2 ,

According to Theorem 8 both choices of the weights in (38) insure that the infinite dimensional integration problem is QMC tractable and the corresponding $\varepsilon$-exponent is no greater than 2. From the proof of this theorem the root mean square discrepancy of the random sample decays like $\mathrm{O}\left(N^{-1 / 2}\right)$, as shown in both Figures 1 and 2. The discrepancy of the Halton sequence in Figure 1 decays roughly like $\mathrm{O}\left(N^{-1 / 2}\right)$ for small $N$ and at a faster rate for large $N$. It would seem that 


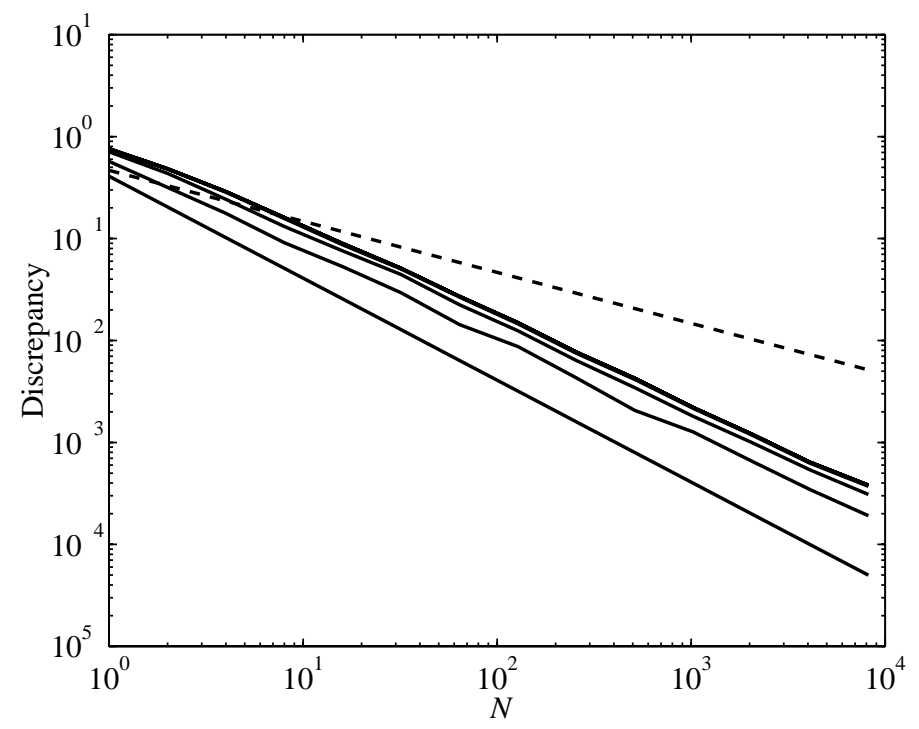

Figure 2. The same as in Figure 1, but for the choice of weights (38b).

the discrepancy for the Halton sequence decays at best like $\mathrm{O}\left(N^{-1 / 2}\right)$ for weights (38a) and $s \rightarrow \infty$. For the choice of weights in (38b), Theorem 9 guarantees that the infinite dimensional Halton sequence has a discrepancy that decreases nearly like $\mathrm{O}\left(N^{-1}\right)$. The plot in Figure 2 is consistent with this conclusion. In fact, the discrepancy in this figure approaches the infinite dimensional case quite quickly; the cases $s=8, \ldots, 128$ are indistinguishable to the eye.

\section{ACKNOWLEDGMENTS}

The authors would like to thank Kai-Tai Fang, Regina H. S. Hong, Ian H. Sloan and Henryk Woźniakowski for their valuable comments and suggestions.

\section{REFERENCES}

[Aro50] M. Aronszajn, Theory of reproducing kernels, Trans. Amer. Math. Soc. 68 (1950), 337404. MR 14:479c

[CMO97] R. E. Caflisch, W. Morokoff, and A. Owen, Valuation of mortgage backed securities using Brownian bridges to reduce effective dimension, J. Comput. Finance 1 (1997), $27-46$.

[DR84] P. J. Davis and P. Rabinowitz, Methods of numerical integration, Academic Press, Orlando, Florida, 1984. MR 86d:65004

[Duf96] D. Duffie, Dynamic asset pricing theory, Princeton University Press, Princeton, New Jersey, 1996.

[Fau82] H. Faure, Discrépance de suites associées à un système de numération (en dimension s), Acta Arith. 41 (1982), 337-351. MR 84m:10050

[FW94] K. T. Fang and Y. Wang, Number-theoretic methods in statistics, Chapman and Hall, New York, 1994. MR 95g:65189

[Hal60] J. H. Halton, On the efficiency of certain quasi-random sequences of points in evaluating multi-dimensional integrals, Numer. Math. 2 (1960), 84-90. MR 22:12688

[Hic98] F. J. Hickernell, A generalized discrepancy and quadrature error bound, Math. Comp. 67 (1998), 299-322. MR 98c:65032

[HW00] F. J. Hickernell and H. Woźniakowski, Integration and approximation in arbitrary dimensions, Adv. Comput. Math. 12 (2000), 25-58. MR 2001d:65017 
[MC94] W. J. Morokoff and R. E. Caflisch, Quasi-random sequences and their discrepancies, SIAM J. Sci. Comput. 15 (1994), 1251-1279. MR 95e:65009

[Nie92] H. Niederreiter, Random number generation and quasi-Monte Carlo methods, CBMSNSF Regional Conference Series in Applied Mathematics, SIAM, Philadelphia, 1992. MR 93h:65008

[NW00] E. Novak and H. Woźniakowski, When are integration and discrepancy tractable?, Foundations of Computational Mathematics (R. De Vore, A. Iserles, E. Süli, eds.), Chap. 8, Cambridge University Press, Cambridge, 2001.

[NX96] H. Niederreiter and C. Xing, Quasirandom points and global function fields, Finite Fields and Applications (S. Cohen and H. Niederreiter, eds.), London Math. Society Lecture Note Series, no. 233, Cambridge University Press, 1996, pp. 269-296. MR 97j:11037

[Sai88] S. Saitoh, Theory of reproducing kernels and its applications, Longman Scientific \& Technical, Essex, England, 1988. MR 90f:46045

[SJ94] I. H. Sloan and S. Joe, Lattice methods for multiple integration, Oxford University Press, Oxford, 1994. MR 98a:65026

[Sob69] I. M. Sobol', Multidimensional quadrature formulas and Haar functions (in Russian), Izdat. "Nauka", Moscow, 1969. MR 54:10952

[Sob98] I. M. Sobol', On quasi-Monte Carlo integration, Math. Comput. Simulation 47 (1998), 103-112. MR 99d:65017

[SW98] I. H. Sloan and H. Woźniakowski, When are quasi-Monte Carlo algorithms efficient for high dimensional integrals, J. Complexity 14 (1998), 1-33. MR 99d:65384

[TW98] J. F. Traub and A. G. Werschulz, Complexity and information, Cambridge University Press, Cambridge, 1998. MR 2000m:65170

[Wah90] G. Wahba, Spline models for observational data, SIAM, Philadelphia, 1990. MR 91g:62028

[Woź91] H. Woźniakowski, Average case complexity of multivariate integration, Bull. Amer. Math. Soc. 24 (1991), 185-194. MR 91i:65224

[WW96] G. W. Wasilkowski and H. Woźniakowski, On tractability of path integration, J. Math. Phys. 37 (1996), 2071-2087. MR 97a:65010

Department of Mathematics, Hong Kong Baptist University, Kowloon Tong, Hong KONG SAR, CHINA

E-mail address: fred@hkbu.edu.hk

Department of Mathematical Sciences, Tsinghua University, Beijing 100084, China

E-mail address: xwang@math.tsinghua.edu.cn 